\title{
Factors Associated with Excessive Daytime Sleepiness in Obstructive Sleep Apnea Syndrome under CPAP Treatment
}

\author{
Wataru Yamadera ${ }^{1,2 *}$, Shintaro Chiba ${ }^{2,3}$, Masayuki Iwashita ${ }^{1}$, Ryo Aoki ${ }^{1}$, Daisuke Harada ${ }^{1}$, \\ Miki Sato $^{1,4}$, Hiroto Moriwaki ${ }^{2}$, Keita Obuchi ${ }^{1}$, Motohiro Ozone ${ }^{1}$, Seiji Nishino ${ }^{4}$, Hiroshi Itoh ${ }^{1}$, \\ Kazuhiko Nakayama ${ }^{1}$
}

${ }^{1}$ Department of Psychiatry, Jikei University School of Medicine, Tokyo, Japan; ${ }^{2}$ Stanford Sleep and Circadian Neurobiology Laboratory, Department of Psychiatry and Behavioral Sciences, School of Medicine, Stanford University, Stanford, USA; ${ }^{3}$ Department of Otorhinolaryngology, Jikei University School of Medicine, Tokyo, Japan; ${ }^{4}$ Shinbashi Sleep Mental Clinic, Tokyo, Japan. Email: ${ }^{*}$ wata-yam@jikei.ac.jp

Received January $4^{\text {th }}, 2012$; revised February $13^{\text {th }}, 2012$; accepted March $24^{\text {th }}, 2012$

\begin{abstract}
The purpose of this study was to assess factors associated with subjective sleep evaluation, chiefly excessive daytime sleepiness (EDS) in obstructive sleep apnea syndrome (OSAS) adult outpatients under continuous positive airway pressure (CPAP) treatment. One thousand and forty-eight OSAS outpatients (mean age: $51.4 \%$ male: $90.5 \%$ ) who were treated by CPAP were consecutively collected. Age, sex, CPAP compliance (CPAP usage as their device of nights with application-time of at least 4 hours per night objectively; \%usage $\geq 4 \mathrm{~h} / \mathrm{d}$ ), and Japanese version of the Pittsburgh Sleep Quality Index (PSQI-J) of the patients showing EDS (Japanese version of the Epworth Sleepiness Scale; JESS $\geq 11$ ) were compared cross-sectionally with those of the patients who did not show EDS (JESS $<11$ ). Nineteen point two \% of all patients showed EDS subjectively. Two hundred one patients were classified to an EDS(+) group and an 847 patients were classified to EDS(-) group. Age and global PSQI-J scores were significantly different between the two groups. Logistic regression showed that EDS was significantly associated with global PSQI-J scores, but not with age. Among PSQI-J components, overall sleep quality, duration of sleep, sleep disturbance, and day dysfunction due to sleepiness were significantly higher in the EDS(+) group. Especially, 19.4\% of patient in the EDS(+) group reported actual sleep time during the past month to be less than 5 hours/day. Although functional relationship should be further evaluated, insufficient sleep is the main factor associated with EDS in the OSAS patients under CPAP treatment.
\end{abstract}

Keywords: Obstructive Sleep Apnea Syndrome; Continuous Positive Airway Pressure; Excessive Daytime Sleepiness; Japanese Version of the Pittsburgh Sleep Quality Index (PSQI-J); Behavioral Induced Insufficient Sleep Syndrome

\section{Introduction}

Continuous positive airway pressure (CPAP) is the firstline treatment of moderate to severe obstructive sleep apnea syndrome (OSAS). The 5-year cumulative survival rates by cardiovascular disease were significantly lower in patients who did not use CPAP than in those who used the device for $>6$ hours/day and 1 to 6 hours/day [1]. It is undoubtedly evident that CPAP treatment for severe OSAS reduces the risk of fatal cardiovascular events [1,2]. CPAP is an effective treatment for OSAS for sleep and physical symptoms' associated with OSAS.

Effective compliance (time spent at the effective pressure) with CPAP in OSAS patients has been reported to

"Corresponding author. be poor. Adequate compliance with CPAP was defined roughly as using the device for at least 4 hours 5 nights a week $[3,4]$, the recommended standards for CPAP compliance to reduce the incidence of cardiovascular diseases $[1,4]$. On the other hand, any standard management, especially for the long-term management to improve excessive daytime sleepiness (EDS) determined by daytime performance and cognition does not exist. Recent metaanalyses demonstrated that CPAP elicited only small improvements in subjective sleepiness in mild to moderate OSAS, and the effects on objective sleepiness are of limited clinical significance [5]. Another meta-analysis showed that CPAP therapy does not improve general QOL scores associated with EDS, but does improve physical domains and vitality [6]. 
Clinically, there are many patients who despite a significant reduction in sleep disordered breathing with good CPAP compliance show little improvement in their EDS. EDS is an important warning to the individual to stop operating because it is dangerous and life-threatening to continue without sleep, especially at risky workpalces [7]. It is important to evaluate factors other than the managements of sleep related breathing disorders and to manage the EDS caused by factors. We therefore evaluated factors associated with subjective sleep evaluation, chiefly EDS in OSAS adult outpatients under CPAP treatment.

\section{Methods}

\subsection{Study Participants and Design}

One thousand and forty-eight adult OSAS outpatients under CPAP treatment by objective monitoring regularly were consecutively collected among those regularly followed at Ohta Memorial Sleep Center, Kawasaki (Table 1). Every patient in whom OSAS was diagnosed went through full standard polysomnography (PSG) [8] in Jikei University School of Medicine, Tokyo or Ohta Memorial Sleep Center, Kawasaki followed by the International Classification of Sleep Disorders $2^{\text {nd }}$ edition (ICSD-2) criteria [9]. Once the diagnosis of moderate to severe OSAS (apnea-hypopnea index $\geq 20$ ) had been established, CPAP was titrated manually with full standard PSG [8]. It is generally accepted that CPAP compliance is satisfactory when the patient uses the device more than $4.5 \mathrm{~h}$ per night [10]. The authors defined good compliance on CPAP usage as their device for at least $70 \%$ of nights with application-time of at least 4 hours per night (\%usage $\geq 4 \mathrm{~h} / \mathrm{d}$ ) by objective measurements [11,12] and adopted as the parameter of CPAP compliance.

In order to investigate the cross sectional relationship between CPAP compliance and subjective sleep evaluation, the authors assessed for EDS using the Japanese version of the Epworth Sleepiness Scale (JESS) [13], and for sleep quality and quantity using the Japanese version of the Pittsburgh Sleep Quality Index (PSQI-J) [14]. The

Table 1. Demographic variables of 1048 patients under CPAP treatment.

\begin{tabular}{ll}
\hline Age (y, [range]) & $51.4 \pm 12.0[23-86]$ \\
\hline Sex (M:F, [\%male]) & $948: 100[90.5]$ \\
\%usage $\geq 4$ h/d (\%, [range]) & $69.1 \pm 28.3[0.0-100.0]$ \\
Global PSQI-J scores [range] & $5.3 \pm 2.9[0-19]$ \\
JESS points [range] & $6.5 \pm 4.8[0-24]$ \\
\hline
\end{tabular}

mean \pm SD or $\mathrm{N} ; \%$ usage $\geq 4 \mathrm{~h} / \mathrm{d}$ : CPAP usage as their device of nights with application-time of at least 4 hours per night by objective measure- ments; PSQI-J: the Japanese version of the Pittsburgh Sleep Quality Index; JESS: the Japanese version of the Epworth Sleepiness Scale. authors categorized JESS total scores rating over 11 as the existence of EDS [13]. PSQI-J were compared with each component, 1) overall sleep quality (SLPQUAL); 2) sleep latency (LATEN); 3) duration of sleep (DURAT); 4) sleep efficacy (HSE); 5) sleep disturbance (DISTB); 6) needed medications to sleep (MEDS), and 7) day dysfunction due to sleepiness (DAYDYS). Each component was rated from 3 to 0 , with global PSQI-J scores rating from 21 to 0 [14].

All patients had check-ups every month after the initiation of CPAP treatment at Ohta Memorial Sleep Center, Kawasaki. In those appointments, from February $1^{\text {st }}$ to April $30^{\text {th }}$ in 2008, patients fulfilled JESS and PSQI-J with the assessment and monitoring objectively for CPAP use. The average use of CPAP in minutes was calculated, as was use efficacy, which refers to the proportion of time the mask was on relative to the total time the CPAP device power was on. Age, sex, \%usage $\geq 4 \mathrm{~h} / \mathrm{d}$, and PSQI-J of the patients showing EDS (JESS $\geq 11$ ) were compared with those of the patients who did not show EDS (JESS < $11)$, in all 1048 patients.

\subsection{Statistical Analysis}

Data were analyzed using Stat View-J5.0 for Windows [SAS Institute Inc.]. Each parameter was compared between the two groups using the unpaired $\mathrm{T}$ test or $\chi^{2}$ test. Logistic regression analysis was conducted to examine subjective excessive daytime sleepiness [EDS(+) or EDS(-)], with age, sex, \%usage $\geq 4 \mathrm{~h} / \mathrm{d}$ and global PSQI-J scores as independent variables. Statistical significance was determined at $\mathrm{P}<0.05$.

\subsection{Approval of the Study}

The study protocol was approved by the Institutional Review Boards of Ohta General Hospital. Written informed consents to participate in the study were obtained from all the participants after they were given an explanation of the study and its potential risks. All of the procedures were carried out in accordance with Good Clinical Practice, the Helsinki Declaration, and related laws.

\section{Results}

Nineteen point two \% of all patients showed EDS subjectively. Two hundred one patients were classified to an EDS(+) group and an 847 patients were classified to EDS(-) group. Age and global PSQI-J scores were significantly different between the two groups (Table 2).

Logistic regression showed that global PSQI-J scores but not age, sex and \%usage $\geq 4 \mathrm{~h} / \mathrm{d}$ significantly influenced the manifestation of EDS in an independent manner (Table 3).

In PSQI-J components, overall sleep quality (C1), duration of sleep (C3), sleep disturbance (C5), and day dys- 
function due to sleepiness (C7) were significantly different between the two groups and were worse in $\operatorname{EDS}(+)$ group (Table 4). The patients of EDS(+) group showed lower subjective sleep quality $(\mathrm{P}<0.001)$, shorter sleep duration $(\mathrm{P}<0.001)$, more sleep disturbed $(\mathrm{P}<0.001)$ and more daytime dysfunction $(\mathrm{P}<0.001)$ significantly compared with those of EDS(-) group.

Distributions of actual sleep time during the past month (PSQI-J, C3) between the $\operatorname{EDS}(+)$ and $\operatorname{EDS}(-)$ group were compared and the results are displayed with a pie graph (Figure 1). There is a clear tendency that percentage of shorter hour sleep is high and that of longer hour sleep is low in the EDS(+) group compared to the EDS(-) group. Especially, $19.4 \%$ of patients $(n=38)$ in the $\operatorname{EDS}(+)$ group reported actual sleep time during the past month to be less than 5 hours/day and this percentage was significantly higher than that of $\operatorname{EDS}(-)$ group $(8.5 \%)$.

Table 2. The Comparison of Patients Characteristics, PSQI-J between the EDS(+) and EDS(-) grou.

\begin{tabular}{lccc}
\hline & $\begin{array}{c}\operatorname{EDS}(+) \\
\text { JESS } \geq 11 \\
(\mathrm{n}=201)\end{array}$ & $\begin{array}{c}\operatorname{EDS}(-) \\
\text { JESS }<11\end{array}$ & \\
& $14.3 \pm 0.2$ & $4.7 \pm 0.1$ & P value \\
\hline JESS points & $49.3 \pm 0.8$ & $52.0 \pm 0.4$ & 0.004 \\
Age (y) & 94.0 & 89.6 & 0.075 \\
Sex (\%male) & $66.0 \pm 1.0$ & $69.9 \pm 2.2$ & 0.077 \\
\%usage $\geq 4$ h/d (\%) & $7.1 \pm 0.2$ & $4.9 \pm 0.1$ & $<0.001$ \\
\hline Global PSQI-J scores & & & \\
\hline
\end{tabular}

Mean \pm SE P value: the unpaired T test or $\chi^{2}$ test, significant difference: $\mathrm{P}<$ 0.05; EDS: excessive daytime sleepiness; \%usage $\geq 4 \mathrm{~h} / \mathrm{d}$ : CPAP usage as their device of nights with application-time of at least 4 hours per night by objective measurements; PSQI-J: the Japanese version of the Pittsburgh Sleep Quality Index; JESS: the Japanese version of the Epworth Sleepiness Scale.
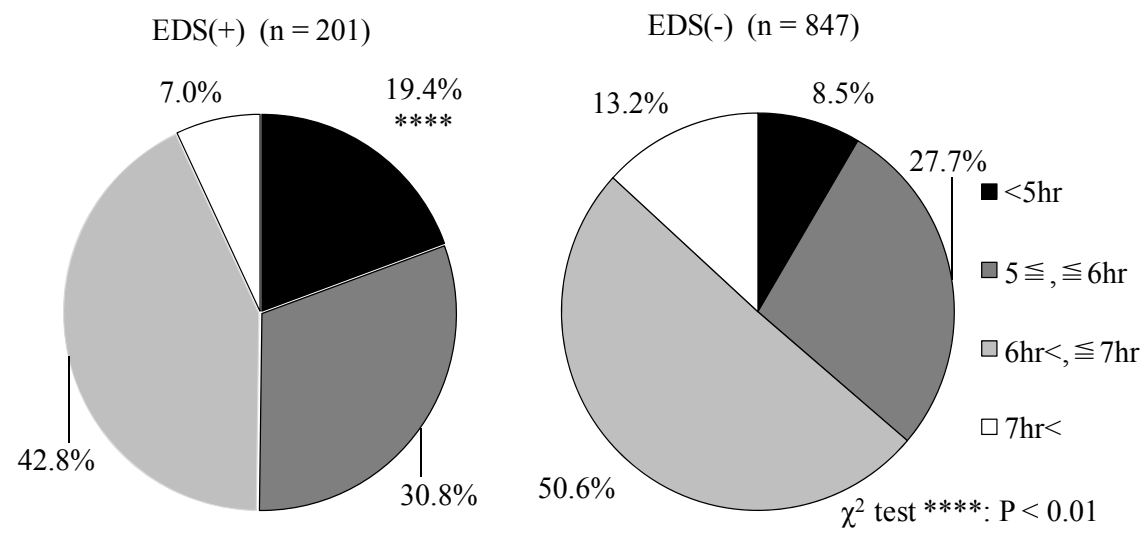

During the past month, how many hours of actual sleep did you get at night? (This may be different than the number of hours you spent in bed.); PSQI-J: the Japanese version of the Pittsburgh Sleep Quality Index; EDS: excessive daytime sleepiness

Figure 1. The comparison of distributions of actual sleep time during the past month (PSQI-J, C3) between the EDS(+) and EDS(-) group. 


\section{Discussion}

We found the high prevalence of EDS in the Japanese adult OSAS patients under CPAP treatment, and nearly $20 \%$ of all patients showed EDS subjectively. This appearance is high compared with previous study in the USA [15]. By subjective sleep evaluations, we found that the global PSQI-J scores were significantly higher in the adult OSAS patients under CPAP treatment with EDS than in those without EDS. In CPAP treated patients who continue to experience EDS, improving CPAP compliance is the first-line strategy [16]. We however, did not find any difference in CPAP compliance judged from \%usage $\geq 4 \mathrm{~h} / \mathrm{d}$ in the two groups. It is also well recognized that some patients continue to experience EDS even after appropriate CPAP treatment [17]. Therefore, it is important to evaluate other factors influence occurrence of EDS in CPAP treated OSA patients.

In PSQI-J component scores in the present study, overall sleep quality (C1), duration of sleep (C3), sleep disturbance (C5), and day dysfunction due to sleepiness (C7) were significantly higher in the EDS(+) group than in the EDS(-) group. It was obvious that day dysfunction due to sleepiness $(\mathrm{C} 7)$ were more severe in the patients with EDS compared to those without EDS. It was suggested that EDS was associated with low subjective sleep quality, short sleep duration and more sleep disturbed in the patients of EDS(+) group. EDS in OSAS is not always caused by sleep apnea alone, although causative relations should further be evaluated. It should be pointed out that sleepiness is a common symptom in the general population, and often results from sleep deprivation. This type of sleepiness will not improve with CPAP if they do not have sleep disorders breathing. EDS in OSAS under good CPAP compliance may be caused clinically, by 1) development of new conditions associated with OSAS/ CPAP, such as increase in weight, rhinitis or other medical illness; or 2) an undiagnosed associated condition such as poor sleep hygiene, treatment with sedating drugs, depression, or other sleep disorders; or 3) loss of placebo (honeymoon) effect revealing the conditions not previously diagnosed [10]. It was suggested that the subjective sleep evaluation, chiefly EDS, in the OSAS patients under CPAP treatment may have involved many factors surrounding the sleep habits, chiefly behaviorally induced insufficient sleep.

\subsection{Behavioral Induced Insufficient Sleep in OSAS Patients}

A common cause of EDS in OSAS patients is insufficient sleep [10], and sleep sufficiency needs to be evaluated in the patients under good CPAP compliance. Use of sleep diaries or actigraph recordings can help in assessing the sleep habits of the patients [10]. When the patient has an unusually high sleep efficacy and/or reports about 2 hours more sleep on each weekend day than each weekday, chronic sleep restriction is suspected [7]. Sleeping with the CPAP more hours will presumably result in improvements of sleepiness. According to the 2010 Nippon Hoso Kyokai (NHK) Japanese Time Use Survey [18], average sleep time per day in adult employed Japanese were 6 hours 55 minutes on weekdays, 7 hours 29 minutes on Saturday, and 7 hours 51 minutes on Sunday. Consequently, each day of the week marked the shortest sleeping hours since 1970. In a cross-sectional self-administered questionnaire survey [19], the 1-month point prevalence of poor sleep quality in Japanese white-collar daytime employees was significantly higher than in the general population of Japanese adults. Most of the OSAS patients treated by CPAP are daytime employee. The present study estimated $19.4 \%$ of the OSAS patients with EDS showed actual sleep time to be less than 5 hours/day. This duration might be equivalent to that of subjects with behaveioral induced insufficient sleep syndrome in ICSD-2 [9]. For behavioral induced insufficient sleep patients, regularizing bedtime and increasing time in bed produces a resolution of their symptoms, but no other manipulations help significantly $[7,20]$.

\subsection{Managements of EDS in the OSAS Patients under CPAP Treatment}

Although our result pointed out that insufficient sleep is one of the most important factors affect EDS in OSAS patients, the management of EDS in the OSAS patients under CPAP treatment is a multifaceted problem including treatment, social and healthcare related factors, and these need to be discuss comprehensively. Knowledge about facilitators and barriers for adherence to CPAP treatment can be used in interventional strategies [21]. This can be increased by intensive patient education. The use of a wake-promoting medication, modafinil, is also approved for OSAS patients who are adherent to CPAP therapy but exhibit a residual EDS [22]. However, the common side effects of modafinil include headache (28\%), anxiety (16\%), and nervousness (14\%). In addition, the possibility that addiction to modafinil may be probable [23]. Before the prescription of stimulants, it is necessary to establish an educational program for OSAS patients under CPAP treatment to enlighten what patients can do for themselves about sleep hygiene.

The limitations of the present study indicated no information about the pretreatment severity of OSAS, changes in body weight and ENT factors, the duration of CPAP use etc. and the use of only two subjective measurements, JESS and PSQI-J. In spite of these limitations, the present study supports the clinical impression that one of the most important and the first thing to manage EDS in pa- 
tients under CPAP treatment is to ensure that their duration of sleep is sufficient.

\section{Disclosure Statement}

This was not an industry supported study. The authors have indicated no financial conflicts of interests.

\section{REFERENCES}

[1] F. Campos-Rodriguez, N. Pena-Grinan, N. Reyes-Nunez, I. Cruz-Moron, J. Perez-Ronchel, F. Vega-Gallardo and A. Fernandez-Palacin, "Mortality in Obstructive Sleep ApneaHypopnea Patients Treated with Positive Airway Pressure," Chest, Vol. 128, No. 2, 2005, pp. 624-633. doi:10.1378/chest.128.2.624

[2] J. M. Marin, S. J. Carrizo, E. Vicente and A. G. Agusti, "LongTerm Cardiovasucular Outcomes in Men with Obstructive Sleep Apnea-Hypopnea with or without Treatment with Continuous Positive Airway Pressure: An Observational Study," Lancet, Vol. 365, No. 9464, 2005, pp. 1046-1053.

[3] N. B. Kribbs, A. I. Pack, L. R. Kline, P. L. Smith, A. R. Schwartz, N. M. Schubert, S. Redline, J. N. Henry, J. E. Getsy and D. F. Dinges, "Objective Measurement of Patterns of Nasal CPAP Use by Patients with Obstructive Sleep Apnea," American Journal of Respiratory and Critical Care Medicine, Vol. 147, No. 4, 1993, pp. 887-895.

[4] M. Orth, S. Kotterba, J. W. Walther, K. Rasche, G. SchultzeWerninghaus and H. W. Duchna, "Long Term Compliance of CPAP-Therapy-Update, Predictors and Interventions," Pneumologie, Vol. 60, No. 8, 2006, pp. 480-484. doi:10.1055/s-2006-944234

[5] N. S. Marshall, M. Barnes, N. Travier, A. J. Campbell, R. J. Pierce, R. D. McEvoy, A. M. Neill and P. H. Gander, "Continuous Positive Airway Pressure Reduces Daytime Sleepiness in Mild to Moderate Obstructive Sleep Apnea: A Meta-Analysis," Thorax, Vol. 61, No. 5, 2006, pp. 430434. doi:10.1136/thx.2005.050583

[6] J. Jing, T. Huang, W. Cui and H. Shen, "Effect on Quality of Life of Continuous Positive Airway Pressure in Patients with Obstructive Sleep Apnea Syndrome: A MetaAnalysis," Lung, Vol. 186, No. 3, 2008, pp. 131-144. doi:10.1007/s00408-008-9079-5

[7] T. Roehrs, M. Carscadon, W. C. Dement and T. Roth, "Daytime Sleepiness and Alertness," In: M. H. Kryeger, T. Roth and W. C. Dement, Eds., Principles and Practice of Sleep Medicine, 5th Edition, Elsevier Sanders, Philadelphia, 2011, pp. 42-53. doi:10.1016/B978-1-4160-6645-3.00004-9

[8] D. Hailey, K. Tran, R. Dales, S. Mensinkai and L. McGahan, "Recommendations and Supporting Evidence in Guideline for Referral of Patients to Sleep Laboratories," Sleep Medicine Review, Vol. 10, No. 4, 2006, pp. 287-299. doi:10.1016/j.smrv.2005.10.004

[9] American Academy of Sleep Medicine, "The International Classification of Sleep Disorders 2nd Edition (ICSD-2), Diagnostic and Coding Manual," American Academy of Sleep Medicine, Westchester, 2005.

[10] J. Santamaria, A. Iranzo, J. Ma Montserrat and J. Pablo, "Persistent Sleepiness in CPAP Treated Obstructive Sleep
Apnea Patients: Evaluation and Treatment," Sleep Medicine Review, Vol. 11, No. 3, 2007, pp. 195-207. doi:10.1016/j.smrv.2007.02.005

[11] H. M. Engleman, S. E. Martin, I. J. Deary and N. J. Douglas, "Effect of Continuous Positive Airway Pressure Treatment on Daytime Function in Sleep Apnea/Hypopnea Syndrome," Lancet, Vol. 343, No. 8897, 1994, pp. 572575. doi:10.1016/S0140-6736(94)91522-9

[12] J. L. Perin, J. Krieger, D. Rodenstein, A. Cornette, E. Sforza, P. Delguste, C. Deschaux, V. Grillier and P. Levy, "Effectiveness Compliance during the First 3 Months of Continuous Positive Airway Pressure: A European Prospective Study of 121 Patients," American Journal of Respiratory and Critical Care Medicine, Vol. 160, No. 4, 1999, pp. 1124-1129.

[13] M. Takegami, Y. Suzukamo, T. Wakita, H. Noguchi, K. Chin, H. Kadotani, Y. Inoue, Y. Oka, T. Nakamura, J. Green, M. W. Johns and S. Fukuhara, "Development of a Japanese Version of the Epworth Sleepiness Scale (JESS) Based on Item Response Theory," Sleep Medicine, Vol. 10, No. 5, 2009, pp. 556-565. doi:10.1016/j.sleep.2008.04.015

[14] Y. Doi, M. Minowa, M. Uchiyama, M. Okawa, K. Kim, K. Shibui and Y. Kamei, "Psychometric Assessment of Subjective Sleep Quality Using the Japanese Version of the Pittsburgh Sleep Quality Index (PSQI-J) in Psychiatric Disordered and Control Subjects," Psychiatry Research, Vol. 97, No. 2, 2000, pp. 165-172. doi:10.1016/S0165-1781(00)00232-8

[15] C. Guilleminault and P. Philip, "Tiredness and Somnolence Despite Initial Treatment of Obstructive Sleep Apnea Syndrome (What to Do When an OSAS Patient Stays Hypersomnolent Despite Treatment)," Sleep, Vol. 19, No. 9S, 1996, pp. 117-122.

[16] M. K. Reeves-Hoche, R. Meck and C. W. Zwillich, "Nasal CPAP, an Objective Evaluation of Patient Compliance," American Journal of Respiratory and Critical Care Medicine, Vol. 149, No. 1, 1994, pp. 149-154.

[17] M. A. Bédard, J. Montplaisir, J. Malo, F. Richer and I. Rouleau, "Persistent Neuropsychological Deficits and Vigilance Impairment in Sleep Apnea Syndrome after Treatment with Continuous Positive Airways Pressure (CPAP)," Journal of Clinical and Experimental Neuropsychology, Vol. 15, No. 2, 1993, pp. 330-341. doi:10.1080/01688639308402567

[18] T. Kobayashi, E. Morofuji and Y. Watanabe, "Sleeping Time Keeps Decreasing, Male Housework Time Is Increasing. From the 2010 NHK Japanese Time Use Survey," The NHK Monthly Report on Broadcast Research, 2011, pp. 2-21.

[19] Y. Doi, M. Minowa and T. Tango, "Impact and Correlates of Poor Sleep Quality in Japanese White-Collar Employees," Sleep, Vol. 26, No. 4, 2003, pp. 467-471.

[20] H. P. Van Dongen, G. Maislin, J. M.Mullington and D. F. Dinges, "The Cumulative Cost of Additional Wakefulness: Dose-Response Effects on Neurobehavioral Functions and Sleep Physiology from Chronic Sleep Restriction and Total Sleep Deprivation," Sleep, Vol. 26, No. 2, 2003, pp. 117-126. 
[21] A. Golay, A. Girard, S. Grandin, J. C. Metrailler, M. Victrion, P. Lebas, J. Ybarra and T. Rochat, "A New Educational Program for Patients Suffering from Sleep Apnea Syndrome," Patient Education and Counseling, Vol. 60, No. 2, 2006, pp. 220-227. doi:10.1016/i.pec.2005.01.007

[22] J. E. Black and M. Hirshkowitz, "Modafinil for Treatment of Residual Excessive Sleepiness in Nasal Continuous Positive Airway Pressure-Treatment Obstructive Sleep
Apnea/Hypopnea Syndrome," Sleep, Vol. 28, No. 4, 2005, pp. 464-471.

[23] W. Charles, Atwood Jr., J. Patrick and R. Givelber, "Medical Therapy for Obstructive Sleep Apnea," In: M. H. Kryeger, T. Roth and W. C. Dement, Eds., Principles and Practice of Sleep Medicine, 5th Edition, Elsevier Sanders, Philadelphia, 2011, pp. 1219-1232. 